\title{
Expression of E-cadherin repressors SNAIL, ZEB1 and ZEB2 by tumour and stromal cells influences tumour-budding phenotype and suggests heterogeneity of stromal cells in pancreatic cancer
}

\author{
J A Galván ${ }^{1}$, I Zlobec ${ }^{1}$, M Wartenberg ${ }^{1,2}$, A Lugli ${ }^{1,2}$, B Gloor ${ }^{3}$, A Perren ${ }^{1,2}$ and E Karamitopoulou ${ }^{\star, 1,2}$ \\ ${ }^{1}$ Translational Research Unit, Institute of Pathology, University of Bern, Murtenstrasse 31, Bern CH-3010, Switzerland; ${ }^{2}$ Division of \\ Clinical Pathology, Institute of Pathology, University of Bern, Murtenstrasse 31, Bern CH-3010, Switzerland and ${ }^{3}$ Department of \\ Visceral Surgery, Insel University Hospital, Freiburgstrasse 4, CH-3010 Bern, Switzerland
}

\begin{abstract}
Background: There is evidence that tumour-stroma interactions have a major role in the neoplastic progression of pancreatic ductal adenocarcinoma (PDAC). Tumour budding is thought to reflect the process of epithelial-mesenchymal transition (EMT); however, the relationship between tumour buds and EMT remains unclear. Here we characterize the tumour-budding- and stromal cells in PDAC at protein and mRNA levels concerning factors involved in EMT.
\end{abstract}

Methods: mRNA in situ hybridisation and immunostaining for E-cadherin, $\beta$-catenin, SNAIL1, ZEB1, ZEB2, N-cadherin and TWIST1 were assessed in the main tumour, tumour buds and tumour stroma on multipunch tissue microarrays from 120 well-characterised PDACs and associated with the clinicopathological features, including peritumoural (PTB) and intratumoural (ITB) budding.

Results: Tumour-budding cells showed increased levels of ZEB1 $(P<0.0001)$ and ZEB2 $(P=0.0119)$ and reduced E-cadherin and $\beta$-catenin $(P<0.0001$, each) compared with the main tumour. Loss of membranous $\beta$-catenin in the main tumour $(P=0.0009)$ and tumour buds $(P=0.0053)$, without nuclear translocation, as well as increased SNAIL1 in tumour and stromal cells $(P=0.0002$, each) correlated with high PTB. ZEB1 overexpression in the main tumour-budding and stromal cells was associated with high ITB $(P=0.0084 ; 0.0250$ and 0.0029 , respectively) and high PTB $(P=0.0005 ; 0.0392$ and 0.0007 , respectively). ZEB2 overexpression in stromal cells correlated with higher pT stage $(P=0.03)$, lymphatic invasion $(P=0.0172)$ and lymph node metastasis $(P=0.0152)$.

Conclusions: In the tumour microenvironment of phenotypically aggressive PDAC, tumour-budding cells express EMT hallmarks at protein and mRNA levels underlining their EMT-type character and are surrounded by stromal cells expressing high levels of the E-cadherin repressors ZEB1, ZEB2 and SNAIL1, this being strongly associated with the tumour-budding phenotype. Moreover, our findings suggest the existence of subtypes of stromal cells in PDAC with phenotypical and functional heterogeneity.

In the era of personalised medicine, pancreatic ductal adenocarcinoma (PDAC) remains a highly lethal malignancy, characterised by a striking desmoplastic reaction. Its lethal nature is attributed to the rapid dissemination of cancer cells to the lymphatic system and distant organs (Hidalgo, 2010; Fernandez-del-Castillo et al, 2013). Moreover, PDAC escapes early detection and resists treatment

*Correspondence: Dr E Karamitopoulou; E-mail: eva.karamitopoulou@pathology.unibe.ch

Received 6 January 2015; revised 16 April 2015; accepted 28 April 2015; published online 19 May 2015

(c) 2015 Cancer Research UK. All rights reserved 0007-0920/15 
(Stathis and Moore, 2010; Fernandez-del-Castillo et al, 2013). To date, however, the management of PDAC remains suboptimal, as available conventional and targeted treatments against the cancer cells have given only limited results. Therefore, the identification of additional prognostic/predictive biomarkers under consideration of the stromal component, which would enable better patient stratification and eventually provide a guide towards a more successful and individualised therapy appears mandatory (Tuveson and Hingorani, 2005; Hidalgo, 2010; Fernandez-delCastillo et al, 2013).

Detachment of cancer cells from the main tumour body and invasion of the surrounding stroma, taking place at the cancerhost interface, is an important first step for the development of distant metastasis (Gabbert et al, 1992). Tumour buds, observed at the invasive front of many gastrointestinal cancers, refer to the presence of isolated single cells or small cell clusters (up to five cells) detached from the main tumour and thought to represent a very aggressive subpopulation of cancer cells (Prall, 2007). Budding cells seem indeed able to degrade the peritumoral connective tissue to evade host's response and finally to infiltrate the lymphatic and blood vessels leading to local and distant metastasis (Lugli et al, 2012). Tumour budding has been consistently associated with adverse clinicopathological characteristics and poor outcome, in most studies independent of other strong prognostic factors such as pathological stage (Hase et al, 1993; Tanaka et al, 2003; Nakamura et al, 2008; Wang et al, 2009). We could recently show that tumour budding is a strong and independent prognostic factor in PDAC (Karamitopoulou et al, 2013a).

Tumour budding is thought to morphologically reflect the process of epithelial-mesenchymal transition (EMT), which is defined in vitro in cell culture models and allows neoplastic epithelial cells to acquire a more mesenchymal phenotype thus increasing their capacity for migration and invasion and helping them become resistant to apoptotic signals (Guarino et al, 2007; Mani et al, 2008; Kalluri, 2009; Thiery et al, 2009; Krantz et al, 2012). However, there is controversy regarding the relationship between budding cells and EMT process, as there is currently no direct evidence that human cancer cells can undergo EMT (Celest et al, 2013). To date, it has not been attempted to further characterize tumour buds; especially no RNA-based studies have been performed.

We hypothesise that tumour-budding cells are EMT-type cells and that the tumour-stroma crosstalk at the tumour microenvironment may influence the EMT-type tumour-budding phenotype in PDAC. Aim of the present study is therefore to characterise the tumour-budding- and stromal cells in pancreatic cancer regarding expression of EMT-related factors at a protein and mRNA level by using mRNA in situ hybridisation (ISH), which has the advantage of preserving the tissue morphology and to correlate the findings with clinicopathologic factors including intratumoral and peritumoral budding (ITB and PTB, respectively). The identification of tumour-budding-promoting profiles could significantly contribute to our understanding of the events that influence progression to invasion, metastasis and drug resistance in PDAC (Arumugam et al, 2009). As most PDAC patients present with advanced disease, resistant to adjuvant therapy, this could also have an implication in future treatment developments.

\section{MATERIALS AND METHODS}

Patients. Overall 120 nonconsecutive PDAC patients with complete treatment and follow-up information, treated at the second Department of Surgery, University of Athens Medical School, were included into the study. Paraffin blocks from all patients have been selected from the archives of the Department of Pathology, University of Athens Medical School, Greece. All selected cases had at least $1.2-\mathrm{cm}$ tumour tissue thickness in at least two paraffin blocks in order to be included into the study. Patients were treated between 2001 and 2011. All histomorphological data were reviewed from the corresponding H\&E-stained slides, whereas clinical data were obtained from corresponding patient records. Clinicopathological data included patient age, gender, tumour location, $\mathrm{pT}, \mathrm{pN}$, $\mathrm{pM}$, lymphatic invasion, vascular invasion, tumour grade, histological subtype, disease-free interval and overall survival. Information on post-operative therapy is available for all patients. Ethical consent has been obtained for this project from the Scientific and Ethical Committee of the Universities of Athens and Bern. Patient characteristics are summarised in Supplementary Table 1. The study design can be visualised in Supplementary Figure 1.

Tissue microarray (TMA) construction. Two TMAs were constructed including punches from primary tumours. In order to exclude bias due to possible tumour heterogeneity, each patient had multiple tumour punches (two from the tumour centre and two from the invasive front, including tumour buds) taken from formalin-fixed, paraffin-embedded blocks using a tissue cylinder with a diameter of $1 \mathrm{~mm}$, which were subsequently transferred into one recipient paraffin block $(3 \times 2.5 \mathrm{~cm})$ using a semiautomated tissue arrayer.

Assessment of tumour budding. Tumour budding was defined as detached single cells or clusters of less than five cells. Cases were evaluated for tumour budding using a 10-in-10 approach (Karamitopoulou et al, 2013b). Briefly, whole-tissue sections of each case underwent immunohistochemistry for AE1/AE3 (pancytokeratin) staining. The 10 densest hotspots of tumour budding were evaluated at high magnification $\left(\times 40,0.55 \mathrm{~mm}^{2}\right)$ and counted. The average number of buds per case was obtained. Although tumour budding is described to occur mostly at the invasive front of cancers (peritumoral budding), in our PDAC series we frequently observed the presence of buds within the main tumour body as well (ITB). Using a receiver operating characteristic (ROC) curve approach, a cutoff score of 10 buds on average was identified as most discriminatory for survival. Cases with an average of $>10$ buds were classified as 'high' budders; those with $\leqslant 10$ buds were assigned as 'low' budders (Supplementary Figure 2).

Immunohistochemical analysis. Whole-tissue sections of formalin-fixed, paraffin-embedded PDAC tissue from all 120 patients have been stained immunohistochemically for $\beta$-catenin (1:500; Abcam, Cambridge, UK), E-Cadherin (1:200; Dako, Glostrup, Denmark), ZEB1 (1:200; Sigma-Aldrich, St Louis, CA, USA), ZEB2 (1:400; Sigma-Aldrich), SNAIL1 (Clone EC3, 1.10; antibody developed and characterised previously by Francí et al, 2006; Mani et al, 2008) N-cadherin (1:300; Abcam), TWIST1 (1:75; Abcam), $\alpha$-smooth muscle actin ( $\alpha$-SMA, 1:100000; Sigma-Aldrich). Antigen retrieval was performed with Tris- $\mathrm{HCl}, \mathrm{pH} 9$ for $30 \mathrm{~min}$ at $95^{\circ}$. Antibody testing and staining protocols have been established and staining was performed by an automated Leica Bond RX System with the Bond Polymer Refine Kit (with DAB as chromogen) and Bond Polymer Refine Red Detection Kit for the double staining (Leica Biosystems, Newcastle, UK). Immunohistochemistry was evaluated by estimating visually the percentage of positive cells per TMA punch in $5 \%$ intervals $(0,5, \ldots, 100 \%)$. In the case of multiple tumour punches per localisation, the average protein expression was calculated across all punches from the same localisation. Evaluation was performed blinded to clinical endpoints. 
mRNA-ISH. mRNA ISH for the EMT genes was performed on TMA sections using the RNAscopeFFPE Assay kit (Advanced Cell Diagnostics, Hayward, CA, USA), on an automated platform (Discovery Ultra, Ventana Medical System - Roche, Tucson, AZ, USA). The following probes from RNA ScopeVR (Advanced Cell Diagnostics) were used: VS Probe-Hs-CDH1, NM_004360.3, probe region: 263-1255; VS Probe-Hs-CTNNB1, NM_0010998210.1, probe region: 285-2315; VS Probe-Hs-SNAI1, NM_005985.3, probe region: 17-1233; VS Probe-Hs-ZEB1, NM_001174096.1, probe region: 548-2253; VS Probe-Hs-ZEB2, NM_001171653.1, probe region: $587-1446$.

As negative control, DapB (dihydrodipicolinate reductase gene) mRNA expression of Bacillus subtilis, and as 'positive control', PPIB (peptidylprolyl isomerase B) were used. A punch core needed to show control positivity to be assessed with the specific RNA probes.

mRNA was found at the cytoplasm of cells and scored as follows: 0 : completely negative; $1+$ : rare dots recognised by high magnification $(\times 40) ; 2+$ : easily recognised dots at high magnification $(40+) ; 3+$ : easily recognised dots at moderate magnification $(\times 20)$; 4 : easily recognised dots at low magnification $(\times 10)$.

Statistical analysis. In order to determine a valid cutoff score for mRNA and/or protein expression (low/high), ROC curve analysis was performed, using the end point of tumour budding. Association of mRNA and/or protein expression with categorical clinicopathological features was performed using the $\chi^{2}$-test and the Fisher's Exact tests; for continuous variables such as age and tumour size, the nonparametric Wilcoxon's Rank Sum test was used. For matched analyses, the Wilcoxon's Signed Rank test for pairs was used. Univariate survival time analysis was performed using the log-rank test and differences plotted using Kaplan-Meier curves. $P$-values $<0.05$ were considered statistically significant. Correction for multiple hypothesis testing was not carried out (Perneger, 1998). Analyses were performed using SAS (V9.2; The SAS Institute, Cary, NC, USA).

\section{RESULTS}

Protein expression of EMT markers. Results are summarised in Table 1. Normal pancreatic tissue and precursor lesions (PanINs) showed a strong membranous staining for E-cadherin and $\beta$-catenin. The low-budding cases also showed preservation of the membranous $\beta$-catenin and E-cadherin and markedly reduced nuclear ZEB1 and ZEB2. On the contrary, high-budding cases showed a reduced membranous expression of both E-cadherin and $\beta$-catenin with almost complete loss of protein expression in the tumour buds. No nuclear translocation of $\beta$-catenin was found. In addition, an increased nuclear ZEB1 and ZEB2 expression was observed in tumours with high budding, especially in the budding cells. Tumour buds frequently expressed cytoplasmic N-cadherin. Nuclear SNAIL1 and TWIST1 expression was seen mostly in the stromal and rarely in some tumour-budding cells. Examples of protein expression of the EMT markers including expression in normal pancreatic tissue, precursor lesions (PanINs) and PDAC specimens are shown in Supplementary Figure 3 (E-cadherin) and Supplementary Figure 4 ( $\beta$-catenin).

Correlation of mRNA levels with EMT marker expression. SNAIL1 mRNA correlated positively with ZEB1 mRNA $(r=0.462)$ and ZEB2 mRNA $(r=0.414)$ as well as with ZEB1 protein expression in the main tumour $(c c 0.631)$ and buds $(r=0.690)$. ZEB1 mRNA showed a negative association with $C D H 1$ (E-cadherin) mRNA $(r=-0.42)$ and CTNNB1 ( $\beta$-catenin) mRNA $(r=-0.326)$, whereas it correlated positively with SNAIL1 mRNA $(r=0.462)$, ZEB2 mRNA $(r=0.455)$ as well as with ZEB1

protein expression in the main tumour $(r=0.371)$, buds $(r=0.333)$ and stromal cells $(r=0.371)$.

ZEB2 mRNA showed a similar negative correlation with $\mathrm{CDH} 1$ mRNA $(r=-0.414)$ and CTNNB1 mRNA $(r=-0.28)$ and a positive correlation with ZEB1 mRNA $(r=0.455)$ and SNAIL1 mRNA $(r=0.414)$. Moreover, it showed surprisingly a negative correlation with ZEB2 protein expression in stromal cells $(r=-0.25)$. CDH1 mRNA showed a strong positive correlation with CTNNB1 mRNA $(r=0.641)$. The results are summarised in Supplementary Table 2. Examples of mRNA-ISH staining of EMT markers including normal pancreatic tissue and PDAC specimens are shown in Supplementary Figure $3(\mathrm{CDH} 1)$ and Supplementary Figure 4; CTNNB1). Positive and negative controls for mRNA-ISH are shown in Supplementary Figure 5.

Differences between the main tumour body and tumour buds. Tumour buds showed a significant loss of membranous E-cadherin and $\beta$-catenin protein expression when compared with the main tumour body $(P<0.0001)$. On the contrary, nuclear ZEB1 and ZEB2 protein expression levels were significantly increased in the tumour buds compared with the main tumour body $(P<0.0001$ and 0.0119 , respectively, Table 2$)$.

Correlation with a tumour-budding phenotype. A high-tumourbudding phenotype, especially regarding PTB, was characterised by loss of membranous $\beta$-catenin in the main tumour and the tumour buds $(P=0.0009$ and 0.0053 , respectively), increased nuclear expression of SNAIL1 in tumour and stromal cells $(P=0.0003$ and 0.0002 , respectively) and increased nuclear expression of ZEB1 in tumour-, budding- and stromal cells $(P=0.0005,0.0392$ and 0.0007 , respectively). Nuclear ZEB2 expression in the buds was also correlated with high PTB $(P=0.0035)$. In addition, loss of membranous E-cadherin in the tumour showed a trend towards high PTB $(P=0.0727$; Table 1$)$.

High ITB was characterised by overexpression of nuclear ZEB1 in the main tumour $(P=0.0084)$, in the tumour buds $(P=0.0250)$ and in the stromal cells $(P=0.0029$; Table 1$)$. Characteristic

Table 1. Correlation of average protein expression of EMT markers with ITB and PTB budding

\begin{tabular}{|l|c|c|c|c|c|c|}
\cline { 2 - 6 } \multicolumn{3}{|c}{} & \multicolumn{5}{c}{ ITB } \\
\cline { 2 - 6 } Markers & Low & High & P-value & Low & High & P-value \\
\hline E-cadherin tumour & 54 & 51.04 & 0.4476 & 54 & 51.4 & 0.4277 \\
\hline E-cadherin buds & 22.4 & 18.32 & 0.1507 & 20.56 & 18.6 & 0.3293 \\
\hline$\beta$-catenin tumour & 36.3 & 39.29 & 0.6233 & 29.26 & 45.26 & 0.0009 \\
\hline$\beta$-catenin buds & 8.75 & 10.04 & 0.8283 & 4.94 & 12.96 & 0.0053 \\
\hline SNAIL tumour & 0.26 & 0.04 & 0.2761 & 0.25 & 0.06 & 0.0002 \\
\hline SNAIL stroma & 4.56 & 5.66 & 0.8173 & 3.06 & 6.81 & 0.0002 \\
\hline SNAIL1 buds & 1 & 1.34 & 0.2883 & 1 & 1.41 & 0.1795 \\
\hline ZEB1 tumour & 20.66 & 30.3 & 0.0084 & 19.42 & 33.6 & 0.0005 \\
\hline ZEB1 buds & 39.33 & 52.7 & 0.0250 & 40.95 & 53.8 & 0.0392 \\
\hline ZEB1 stroma & 41.12 & 51.8 & 0.0029 & 40.73 & 53.5 & 0.0007 \\
\hline ZEB2 tumour & 38.47 & 41.4 & 0.5097 & 47 & 36.6 & 0.1566 \\
\hline ZEB2 buds & 44.74 & 43.3 & 0.8464 & 55.5 & 36.4 & 0.0035 \\
\hline ZEB2 stroma & 1.9 & 1.6 & 0.7454 & 2.22 & 1.55 & 0.6603 \\
\hline N-cadherin tumour & 0.04 & 0.07 & 0.8004 & 0.05 & 0.07 & 0.5855 \\
\hline N-cadherin buds & 0.16 & 0.19 & 0.9081 & 0.22 & 0.16 & 0.4976 \\
\hline TWIST1 tumour & 0.097 & 0.1 & 0.9631 & 0.14 & 0.07 & 0.2261 \\
\hline TWIST1 stroma & 0.32 & 0.33 & 0.9885 & 0.31 & 0.31 & 0.9497 \\
\hline TWIST1 buds & 0.06 & 0.03 & 0.3543 & 0.08 & 0 & 0.0737 \\
\hline Abbreviations: EMT $=$ epithelial-mesenchymal & transition; & ITB $=$ intratumoural & budding; \\
\hline PTB= peritumoural budding. Statistically significant $P$ values are in bold. & \\
\hline & & & & & & \\
\hline
\end{tabular}


examples of protein- and mRNA-ISH staining of the EMT markers in the tumour buds are depicted in Figure 1.

Correlation with clinicopathological features. High levels of SNAIL1 mRNA $(P=0.0142)$ and ZEB1 mRNA $(P=0.0243)$, as well as TWIST protein expression in the tumour buds $(P=0.0023)$, were associated with the presence of distant metastasis (pM1, Supplementary Table 3). ZEB2 protein expression in the tumour stroma was associated with higher $\mathrm{pT}$ stage $(P=0.03)$, lymphatic invasion $(P=0.0172)$ and the presence of lymph node metastasis $(P=0.0152)$. ZEB1 overexpression in the main tumour was associated with positive resection margins

Table 2. Differences on average protein expression between the main tumour and tumour buds

\begin{tabular}{|l|c|c|c|} 
& Main tumour & Buds & $P$-value (matched) \\
\hline E-cadherin & 52.9 & 19.7 & $<0.0001$ \\
\hline$\beta$-catenin & 38 & 9.4 & $<0.0001$ \\
\hline ZEB1 & 25.8 & 46.3 & $<0.0001$ \\
\hline ZEB2 & 30 & 43.7 & 0.0119 \\
\hline
\end{tabular}

(R1, $P=0.0043)$ and $\mathrm{N}$-cadherin overexpression with higher the T-stage $(P=0.0194$; Supplementary Table 3$)$.

ZEB1 and ZEB2 expression in stromal cells. Increased levels of ZEB1 protein and ZEB1 mRNA were observed not only in the main tumour and in the tumour buds but in the tumour stroma as well. Moreover, ZEB1 expression in the stromal cells was associated with a high-tumour-budding phenotype, whereas ZEB2 positivity was mostly seen in cases with the higher T-stage, lymphatic invasion and presence of lymph node metastases (Supplementary Table 3). Stromal cells surrounding PanIN and stromal cells in non-neoplasic pancreatic tissue remained negative for ZEB1 and/or ZEB2. Examples of mRNA-ISH and ZEB1- and ZEB2-protein expression by the stromal cells, including double staining for $Z E B 1 / \alpha-S M A$ and ZEB2/ $\alpha$-SMA to better demonstrate the nature of ZEB1- and/or ZEB2-positive stromal cells as cancer-associated fibroblasts, are shown in Figure 2. The schematic representation of the most important findings is shown in Figure 3. Expression overlaps between main tumour, tumour buds and stroma are highlighted in Figure 4.
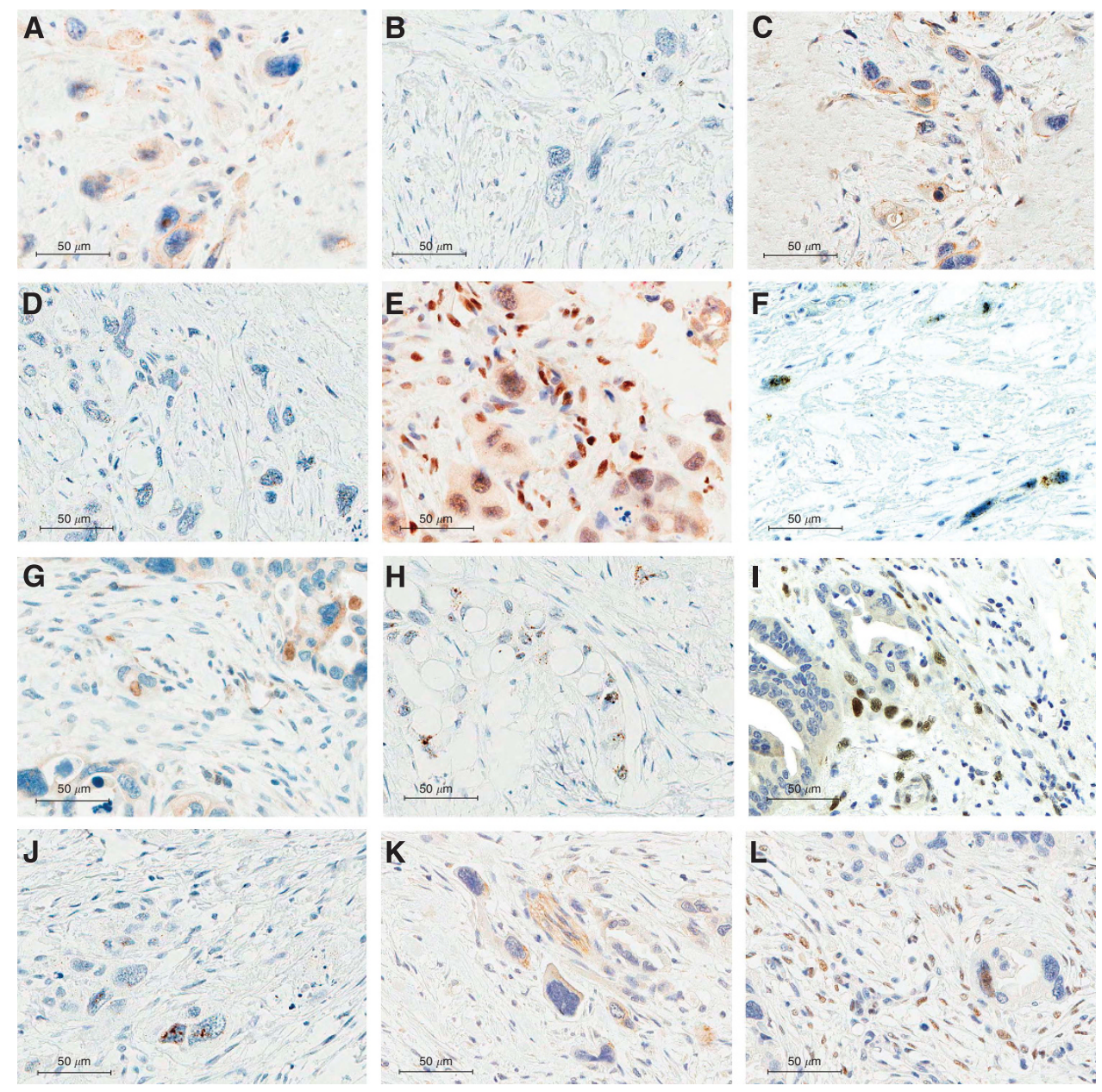

Figure 1. Examples of IHC and mRNA-ISH staining for EMT markers in the tumour buds. (A) Reduced membranous E-cadherin protein expression in the tumour buds, $\times 600$. (B) Reduced signals of CDH1 mRNA in the tumour buds, $\times 600$. (C) Tumour buds with reduced $\beta$-catenin protein expression, $\times 600$. (D) Reduced signals of CTNNB1 mRNA in the tumour buds, $\times 600$. (E) Nuclear expression of the ZEB1 protein in the tumour buds, $\times 600$. (F) Many signals of Zeb1 mRNA in the tumour buds, $\times 600$. (G) Nuclear expression of the ZEB2 protein in the tumour buds, $\times 600$. (H) Many signals of Zeb2 mRNA in the tumour buds, $\times 600$. (I) Strong nuclear expression of the SNAIL1 protein in the tumour buds, $\times 600$. (J) Many signals of Snail1 mRNA in the tumour buds, $\times 600$. (K) Increased N-cadherin protein expression in the budding cells, $\times 600$. (L) Increased TWIST protein expression in the budding cells, $\times 600$. 


\section{DISCUSSION}

In the present study we characterize the tumour-budding- and stromal cells in pancreatic cancer and show that budding cells
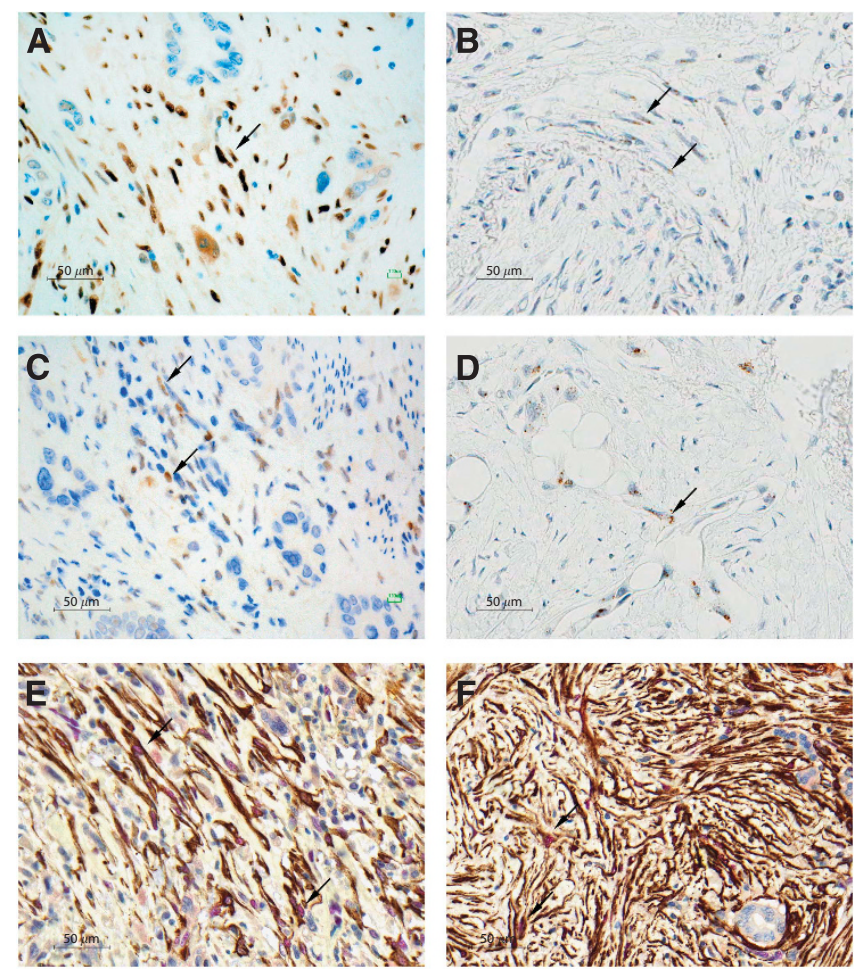

Figure 2. Examples of IHC and mRNA-ISH staining for ZEB1 and ZEB2 in the stromal cells. (A) Strong nuclear expression of the ZEB1 protein in the tumour stroma, $\times 400$. (B) Many signals of Zeb1 mRNA in the stromal cells, $\times 400$. (C) Strong nuclear expression of the ZEB2 protein in the tumour stroma, $\times 400$. (D) Many signals of Zeb2 mRNA in the stromal cells, $\times 400$. (E) Double staining showing nuclear expression of the ZEB1 protein (in red) in $\alpha$-SMA-positive stromal cells (brown),

$\times 400$. (F) Double staining demonstating nuclear expression of the ZEB2 protein (in red) in $\alpha$-SMA-positive cells (brown) in the tumour stroma, $\times 400$. express EMT hallmarks at protein and mRNA levels. Moreover, we find that stromal cells in the immediate environment of tumour buds express the E-cadherin repressors SNAIL1 and ZEB1, this expression being strongly associated with the EMT-type tumourbudding phenotype, whereas the expression of ZEB2 by the stromal cells significantly correlates to the presence of lymph node metastasis.

Although tumour budding has been likened to EMT, the relationship between budding cells and EMT-type cells remains currently unclear. Budding cells examined mainly in gastrointestinal cancers, such as colorectal cancer, have been shown by immunohistochemistry to lack membranous E-cadherin, to express nuclear $\beta$-catenin indicating activation of WNT signalling and to overexpress fibronectin and laminin 5 gamma 2, suggesting an EMT phenotype (Kirchner and Brabletz, 2000; Schmalhofer, et al, 2009). In a recently published study Bronsert et al (2014) performed a quantitative three-dimensional (3D) assessment of tumour budding at the cancer-host interface in human pancreatic, colorectal, lung and breast adenocarcinoma showing that budding tumour cells display a shift towards spindle-like as well as rounded morphology, associated with decreased E-Cadherin-staining intensity and increased nuclear ZEB1 expression. In agreement with this we show that in pancreatic cancer tumour-budding cells not only have an immunohistochemical EMT signature, but this is also reflected by the mRNA levels of the examined EMT factors within the cell cytoplasm. Our findings underline the EMT-type character of budding cells showing that the morphological phenotype of tumour budding is genetically very similar to the EMT process. Especially the combination 'SNAIL1 $+/ \mathrm{ZEB} 1+/ \beta$-catenin - ' correlated strongly with high budding and can be regarded as 'budding-promoting profile' in PDAC.

In contrast to other cancers such as colorectal cancer where budding cells have been described to express nuclear $\beta$-catenin (Kirchner and Brabletz, 2000), this phenotype was not seen in any of our PDAC cases. In the present study tumour-budding cells displayed loss of membranous $\beta$-catenin, paralleled by reduced CTNNB1 mRNA levels, but without cytoplasmic accumulation or nuclear translocation of the protein. This may indicate that the activation of the canonical Wnt pathway is not a prerequisite for the process of EMT-type tumour budding in PDAC. Activation of the $\mathrm{Wnt} / \beta$-catenin pathway in pancreatic cancer has been mainly shown in tissue culture studies (Zhang et al, 2013; Arensman et al, 2014), whereas in the literature there are some contradicting results

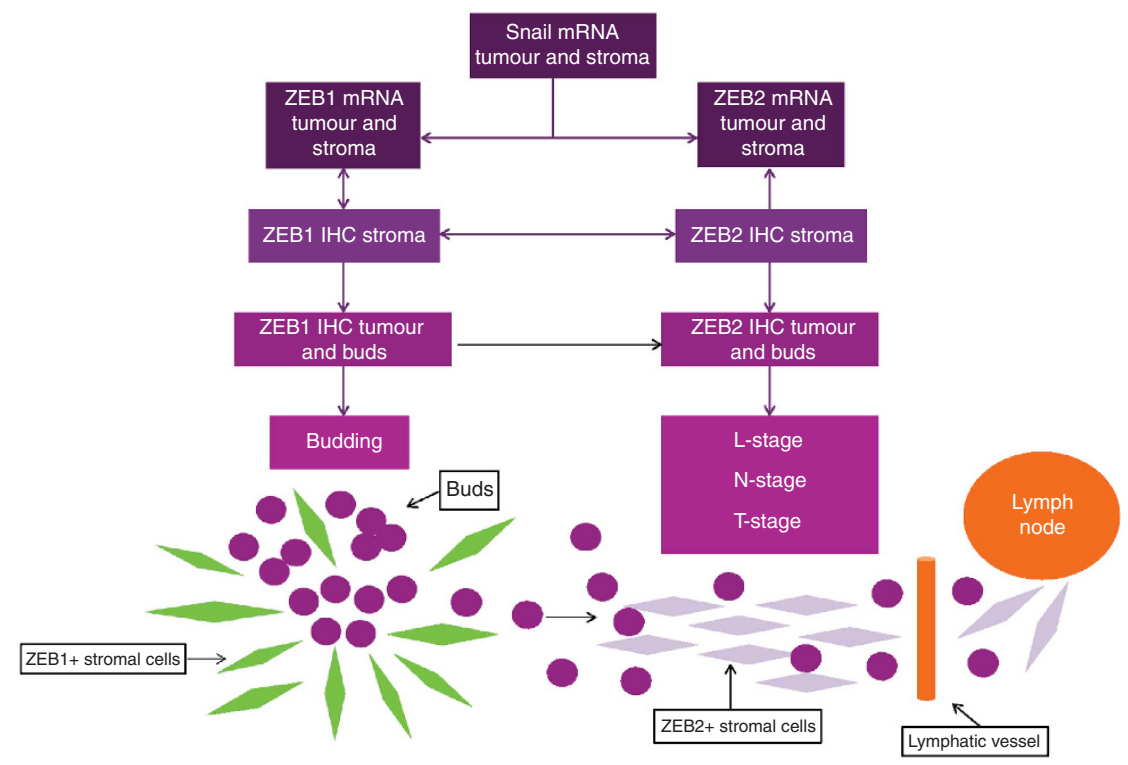

Figure 3. Schematic representation of the most important findings of the present study. 


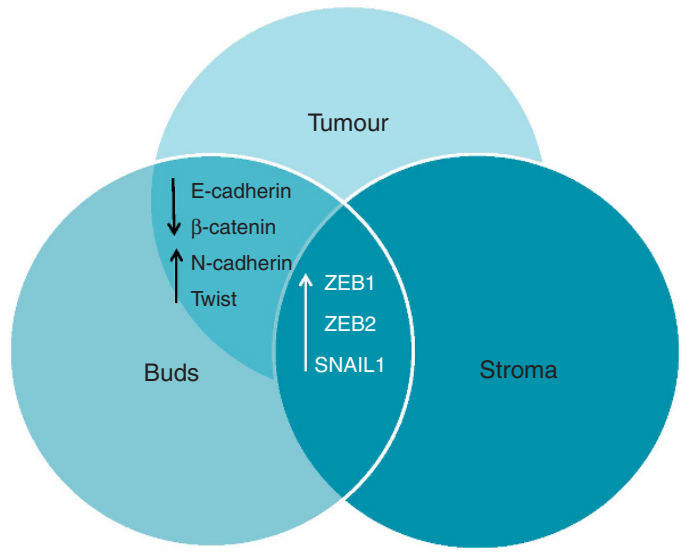

Figure 4. Venn diagram demonstrating expression overlaps between main tumour, tumour buds and stromal cells.

regarding activation of the canonical $v s$ non-canonical Wnt pathway (Weekes and Winn, 2011; Zhang et al, 2013). Moreover, mutations in APC, AXIN or CTNNB1 that hyperactivate Wnt/ $\beta$-catenin signalling are rare in PDAC (White et al, 2012). Our results support the view that in the PDAC canonical Wnt pathway is probably not involved at this advanced stage of cancer cell invasion and migration. Rather the activation of other pathways, known to be involved in pancreatic cancer progression like the TGF $\beta$ pathway (Ellenrieder et al, 2001; Gore et al 2014) may induce the EMT-type tumour-budding phenotype in pancreatic cancer. Indeed, according to previous studies TGFbdriven EMT is sufficient to generate migrating cancer stem cells by directly linking the acquisition of cellular motility with the maintenance of tumour-initiating (stemness) capacity (Cufí et al, 2010). However, a low-level activation of the canonical Wnt pathway that escapes detection by immunohistochemistry cannot be excluded.

There is strong evidence that stromal cells by interacting with tumour cells are involved in pancreatic cancer progression (Apte et al, 2013). Our findings suggest that this may involve the regulation of the EMT-type tumour-budding phenotype and the metastatic spread in PDAC. However, the mechanism of this interaction remains unclear. One hypothesis could be that some stromal cells represent complete mesenchymally transformed tumour cells. Indeed, stromal cells have been found to exhibit similar genetic alterations with tumour cells in chromosomeunstable colorectal cancers (Celesti et al, 2013). Another hypothesis could be that there is a special phenotype of cancer-associated fibroblasts associated with and supporting EMT-type tumour budding through cellular crosstalk in the tumour microenvironment of PDAC. Expression of the transcription factor ZEB1 has been reported to occur in the stromal compartment of breast carcinomas, supposing to represent two populations of cells: EMTtransformed neoplastic cells and stromal fibroblastic cells undergoing activation of ZEB1 due to growth factors produced by the tumour (Soini et al, 2011). Furthermore, Schulte et al (2012) recently reported that distinct subpopulations of fibroblasts are to various extents associated with EMT and tumour progression in urothelial bladder cancer. In accordance to this, our results suggest that there are distinct phenotypes of stromal cells exhibiting different clinicopathological associations. Indeed, the SNAIL1 + 1 ZEB1 + /ZEB2 - phenotype of the stromal cells was associated with high EMT-type tumour budding, whereas the SNAIL1 - I ZEB1 - /ZEB2 + phenotype was mostly seen in cases with higher T-stage, lymphatic invasion and presence of lymph node metastases. Interestingly, these phenotypes were restricted to PDAC tissues and were not found in the normal pancreatic tissue or in the stroma surrounding PanINs.
This study should be understood in the context of its limitations. Although TMAs provide an efficient and cost-effective tool for testing a comprehensive panel of potential biomarkers on a large number of tumour specimens, the TMA technique could raise concerns related to the sampling of large, heterogeneous tumours. The effect of tumour heterogeneity was minimised by sampling at least four punches from each tumour and by especially including punches from the advancing tumour front in order to contain the tumour buds. Our study may further be limited by the fact that all cases come from a single centre. Nonetheless, our study benefits from complete clinicopathological data and the adherence to the REMARK guidelines (McShane et al, 2005).

This study has also several strong points. To our knowledge, this may be the first study to characterize tumour buds at the mRNA level by using mRNA-ISH that has the advantage of preservation of the tissue and cellular morphology. Moreover, we carry out thorough evaluation of mRNA and corresponding protein expression of several EMT markers in the main tumour, the tumour buds and the tumour stroma.

In conclusion, our results show that in the tumour microenvironment of PDAC tumour-budding cells not only express hallmarks of EMT at the protein and mRNA level, but are also surrounded by stromal cells expressing transcription factors that are strongly associated with an EMT-type tumour-budding phenotype. Moreover, our findings suggest that EMT-type tumour budding in pancreatic cancer, in contrast to other cancers, occurs without detectable nuclear $\beta$-catenin suggesting no or low-level activation of the canonical Wnt pathway. Furthermore, our findings support the existence of phenotypically distinct subtypes of cancerassociated fibroblasts in PDAC with different clinicopathological associations.

\section{ACKNOWLEDGEMENTS}

This project was supported by the Johanna Durmüller-Bol Foundation and the Bernese Cancer League.

\section{CONFLICT OF INTEREST}

The authors declare no conflict of interest.

\section{DISCLAIMER}

The funders had no involvement in the study design; in the collection, analysis and interpretation of the data; in the writing of the report; and in the decision to submit the paper for publication.

\section{REFERENCES}

Apte MV, Wilson JS, Lugea A, Pandol SJ (2013) A starring role for stellate cells in the pancreatic cancer microenvironment. Gastroenterology 144 : $1210-1219$.

Arensman MD, Kovochich AN, Kulikauskas RM, Lay AR, Yang PT, Li X, Donahue T, Major MB, Moon RT, Chien AJ, Dawson DW (2014) WNT7B mediates autocrine $\mathrm{Wnt} / \beta$-catenin signaling and anchorage-independent growth in pancreatic adenocarcinoma. Oncogene 33: 899-908.

Arumugam T, Ramachandran V, Fournier KF, Wang H, Marquis L, Abbruzzese JL, Gallick GE, Logsdon CD, McConkey DJ, Choi W (2009) Epithelial to mesenchymal transition contributes to drug resistance in pancreatic cancer. Cancer Res 69: 5820-5828.

Bronsert P, Enderle-Ammour K, Bader M, Timme S, Kuehs M, Csanadi A, Kayser G, Kohler I, Bausch D, Hoeppner J, Hopt UT, Keck T, Stickeler E, Passlick B, Schilling O, Reiss CP, Vashist Y, Brabletz T, Berger J, Lotz J, Olesch J, Werner M, Wellner UF (2014) Cancer cell invasion and EMT 
marker expression - a three-dimensional study of the human cancer-host interface. J Pathol 234: 410-422.

Celesti G, Di Caro G, Bianchi P, Grizzi F, Basso G, Marchesi F, Doni A, Marra G, Roncalli M, Mantovani A, Malesci A, Laghi L (2013) Presence of Twist1positive neoplastic cells in the stroma of chromosome-unstable colorectal tumors. Gastroenterology 145: 647-657.

Cufí S, Vazquez-Martin A, Oliveras-Ferraros C, Martin-Castillo B, Joven J, Menendez JA (2010) Metformin against TGF $\beta$-induced epithelial-tomesenchymal transition (EMT): from cancer stem cells to aging-associated fibrosis. Cell Cycle 9: 4461-4468.

Ellenrieder V, Hendler SF, Boeck W, Seufferlein T, Menke A, Ruhland C, Adler G, Gress TM (2001) Transforming growth factor beta1 treatment leads to an epithelial mesenchymal transdifferentiation of pancreatic cancer cells requiring extracellular signal -regulated kinase 2 activation. Cancer Res 61: 4222-4228.

Fernandez-del-Castillo C, Jimenez RE, Steer ML (2013) Surgery in the treatment of exocrine pancreas and prognosis. Editor: Tanabe KK. www.uptodate.com.

Francí C, Takkunen M, Dave N, Alameda F, Gómez S, Rodríguez R, Escrivà M, Montserrat-Sentís B, Baró T, Garrido M, Bonilla F, Virtanen I, García de Herreros A (2006) Expression of Snail protein in tumor-stroma interface. Oncogene 25: 5134-5144.

Gabbert HE, Meier S, Gerharz CD, Hommel G (1992) Tumor-cell dissociation at the invasion front: a new prognostic parameter in gastric cancer patients. Int J Cancer 50: 202-207.

Gore AJ, Deitz SL, Palam LR, Craven KE, Korc M (2014) Pancreatic cancerassociated retinoblastoma 1 dysfunction enables TGF- $\beta$ to promote proliferation. J Clin Invest 124: 338-352.

Guarino M, Rubino B, Ballabio G (2007) The role of epithelial-mesenchymal transition in cancer pathology. Pathology 39: 305-318.

Hase K, Shatney C, Johnson D, Trollope M, Vierra M (1993) Prognostic value of tumor "budding" in patients with colorectal cancer. Dis Colon Rectum 36: 627-635.

Hidalgo M (2010) Pancreatic cancer. N Engl J Med 362: 1605-1617.

Kalluri R (2009) EMT: when epithelial cells decide to become mesenchymallike cells. J Clin Invest 119: 1417-1419.

Karamitopoulou E, Zlobec I, Born D, Kondi-Pafiti A, Lykoudis P, Mellou A, Gennatas K, Gloor B, Lugli A (2013a) Tumor budding is a strong and independent prognostic factor in pancreatic cancer. Eur J Cancer 49: 1032-1039.

Karamitopoulou E, Zlobec I, Kölzer V, Kondi-Pafiti A, Patsouris ES, Gennatas K, Lugli A (2013b) Proposal for a 10-high-power-fields scoring method for the assessment of tumor budding in colorectal cancer. Mod Pathol 26: 295-301.

Kirchner T, Brabletz T (2000) Patterning and nuclear beta-catenin expression in the colonic adenoma-carcinoma sequence. Analogies with embryonic gastrulation. Am J Pathol 157: 1113-1121.

Krantz SB, Shields MA, Dangi-Garimella S, Munshi HG, Bentrem DJ (2012) Contribution of epithelial-to-mesenchymal transition and cancer stem cells to pancreatic cancer progression. J Surg Res 173: 105-112.

Lugli A, Karamitopoulou E, Zlobec I (2012) Tumour budding: a promising parameter in colorectal cancer. Br J Cancer 106: 1713-1717.

Mani SA, Guo W, Liao M-J, Eaton EN, Ayyanan A, Zhou AY, Brooks M, Reinhard F, Zhang CC, Shipitsin M, Campbell LL, Polyak K, Brisken C,
Yang J, Weinberg RA (2008) The epithelial-mesenchymal transition generates cells with properties of stem cells. Cell 133: 704-715.

McShane LM, Altman DG, Sauerbrei W, Taube SE, Gion M, Clark GM. Statistics Subcommittee of the NCI-EORTC Working Group on Cancer Diagnostics (2005) REporting recommendations for tumour MARKer prognostic studies (REMARK). Br J Cancer 93: 387-391.

Nakamura T, Mitomi H, Kanazawa H, Ohkura Y, Watanabe M (2008) Tumor budding as an index to identify high-risk patients with stage II colon cancer. Dis Colon Rectum 51: 568-572.

Perneger TV (1998) What's wrong with Bonferroni adjustments. Br MedJ 7139: $1236-1238$.

Prall F (2007) Tumour budding in colorectal carcinoma. Histopathology 50: 151-162.

Schmalhofer O, Brabletz S, Brabletz T (2009) E-cadherin, beta-catenin, and ZEB1 in malignant progression of cancer. Cancer Metastasis Rev 28: 151-166.

Schulte J, Weidig M, Balzer P, Richter P, Franz M, Junker K, Gajda M, Friedrich K, Wunderlich H, Östman A, Petersen I, Berndt A (2012) Expression of the E-cadherin repressors Snail, Slug and Zeb1 in urothelial carcinoma of the urinary bladder: relation to stromal fibroblast activation and invasive behaviour of carcinoma cells. Histochem Cell Biol 138: 847-860.

Soini Y, Tuhkanen H, Sironen R, Virtanen I, Kataja V, Auvinen P, Mannermaa A, Kosma VM (2011) Transcription factors zebl, twist and snail in breast carcinoma. BMC Cancer 11: 73-81.

Stathis A, Moore MJ (2010) Advanced pancreatic carcinoma: current treatment and future challenges. Nat Rev Clin Oncol 7: 163-172.

Tanaka M, Hashiguchi Y, Ueno H, Hase K, Mochizuki H (2003) Tumor budding at the invasive margin can predict patients at high risk of recurrence after curative surgery for stage II, T3 colon cancer. Dis Colon Rectum 46: 1054-1059.

Thiery JP, Acloque H, Huang RY, Nieto MA (2009) Epithelial-mesenchymal transitions in development and disease. Cell 139: 871-890.

Tuveson DA, Hingorani SR (2005) Ductal pancreatic cancer in humans and mice. Cold Spring Harb Symp Quant Biol 70: 65-72.

Wang LM, Kevans D, Mulcahy H, O’Sullivan J, Fennelly D, Hyland J, O'Donoghue D, Sheahan K (2009) Tumor budding is a strong and reproducible prognostic marker in T3N0 colorectal cancer. Am J Surg Pathol 33: 134-141.

Weekes CD, Winn RA (2011) The many faces of wnt and pancreatic ductal adenocarcinoma oncogenesis. Cancers 3: 3676-3686.

White BD, Chien AJ, Dawson DW (2012) Dysregulation of Wnt/beta-catenin signaling in gastrointestinal cancers. Gastroenterology 142: 219-232.

Zhang Y, Morris JP, Yan W, Schofield HK, Gurney A, Simeone DM, Millar SE, Hoey T, Hebrok M, Pasca di Magliano M (2013) Canonical wnt signaling is required for pancreatic carcinogenesis. Cancer Res 73: 4909-4922.

This work is published under the standard license to publish agreement. After 12 months the work will become freely available and the license terms will switch to a Creative Commons AttributionNonCommercial-Share Alike 4.0 Unported License.

Supplementary Information accompanies this paper on British Journal of Cancer website (http://www.nature.com/bjc) 